\title{
Design and synthesis of chiral urea-derived iodoarenes and their assessment in the enantioselective dearomatizing cyclization of a naphthyl amide
}

\author{
M. Umair Tariq ${ }^{\mathrm{a}}$ and Wesley $\mathrm{J}$ Moran $^{\mathrm{a}} *$ \\ ${ }^{a}$ Department of Chemistry, University of Huddersfield, Queensgate, Huddersfield HD1 3DH, U.K.
}

\section{ARTICLE INFO}

\section{ABSTRACT}

Article history:

\section{Received}

Received in revised form

Accepted

Available online

\section{Keywords:}

catalysis

hypervalent iodine

iodoarene

dearomatization

cyclization
A novel family of urea-derived chiral iodoarenes was designed and synthesized for use in enantioselective iodine(I/III) catalysis. Their preparation required the development of a bidirectional synthetic strategy. These new chiral iodoarenes were assessed as catalysts in the dearomatizing cyclization of a naphthyl amide and provided moderate yields of product in some cases with low enantioselectivities.

\section{Introduction}

Enantioselective reactions mediated or catalyzed by chiral hypervalent iodine reagents or chiral iodoarene precatalysts have attracted significant attention over the past decade. ${ }^{1}$ In this regard, a variety of chiral iodoarene backbones have been reported; however, the most successful, and most reported, framework is the bislactate ethers $\mathbf{1}$ and $\mathbf{2}$ reported independently by Fujita and Ishihara (Fig. 1). ${ }^{2,3}$ A number of modifications of this skeleton have been investigated, ${ }^{4}$ as each new application often requires a new derivative for optimal results. Other reported chiral iodoarenes include Zhang's spirobiindane derivative 3, ${ }^{5}$ Quideau's helicene 4, ${ }^{6}$ Maruoka's indane 5, Ibrahim's dimethanoanthracene 6, ${ }^{8}$ and Moran's pseudoephedrine derivative $7 .^{9}$ More recently, Nachtsheim reported his second generation triazole catalyst $\mathbf{8}$ which appears to yield superior selectivities in a range of enantioselective oxidation reactions. ${ }^{10}$ It is clear that, as in other areas of enantioselective catalysis, there is no panacea and the investigation of new chiral iodoarenes and hypervalent iodine reagents is still of interest and importance.<smiles>[X]C(=O)[C@H](C)Oc1cccc(O[C@@H](C)C([X])=O)c1I</smiles>

$1 \mathrm{X}=\mathrm{OR}$

$2 \mathrm{X}=\mathrm{NR}_{2}$<smiles>C[C@H](Oc1ccc2c(c1I)[C@H](OCc1ccccc1)C(C)(C)C2)C(=O)Nc1c(Br)cccc1Br</smiles><smiles>CC(C)(C)c1ccc(-c2cc(C(F)(F)F)cc(C(F)(F)F)c2)cc1</smiles>

Fig. 1. Examples of chiral iodoarenes

We recently reported the dearomatizing cyclization of phenols and naphthols with pendent amides catalyzed by iodoarenes (Scheme 1). ${ }^{11}$ We envisaged that the use of chiral iodoarene precatalysts would enable the enantioselective cyclization of the naphthols. 
<smiles>[R]C(=O)NNc1ccc(O)cc1</smiles>

$9 n=1,2$

20-40 mol\% 4-iodotoluene<smiles>[R]c1ccc2c(CNC(=O)c3ccccc3)c(O)ccc2c1</smiles>

1.6 equiv $m$-CPBA

HFIP, rt, 16 hours<smiles>[R]C1=NNC2(C=CC(=O)C=C2)O1</smiles>

10<smiles>[R]c1ccc2c(c1)C=CC(=O)C21CN=C(c2ccccc2)O1</smiles>

12

Scheme 1. Dearomatizing cyclization of phenols and naphthols bearing pendent amides

\section{Results and Discussion}

At the outset of the project we tested the efficacy of the known iodoarenes 1a and 2a on their ability to catalyze the dearomatizing cyclization of naphthol 11a. ${ }^{12}$ After some experimentation with differentwe did weeyes solvents and temperature, the best selectivity observed with 1a was $4 \%$ ee and with $\mathbf{2 a}$ was $14 \%$ ee (Scheme 2). These preliminary observations suggested that the reaction could be rendered enantioselective but that an alternative catalyst structure would probably be required for high levels of enantioinduction.<smiles>O=C(NCc1c(O)ccc2ccccc12)c1ccccc1</smiles>

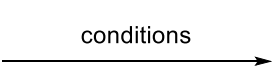

$20 \mathrm{~mol} \% 1 \mathrm{a}, 2.4$ equiv $m$-CPBA, $2: 1 \mathrm{MeCN} / \mathrm{EtOH}, 20{ }^{\circ} \mathrm{C}, 24 \mathrm{~h}$

10 mol\% 2a, 1.2 equiv $m$-CPBA, $3: 1 \mathrm{CH}_{2} \mathrm{Cl}_{2} / \mathrm{MeOH},-20^{\circ} \mathrm{C}, 18 \mathrm{~h}$

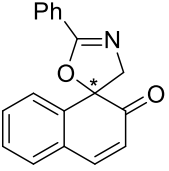

$12 a$<smiles>COC(=O)C(Cc1ccccc1)Oc1cc(C(C)=O)cc(O[C@H](Br)C(=O)OC)c1I</smiles>

Scheme 2. Initial attempts at the dearomatizing cyclization of naphthyl amide 11a

At this juncture we wished to design a new family of chiral iodoarenes for use in iodine(I/III) catalysis and investigate their efficacy in the enantioselective dearomatizing cyclization of naphthols. Specifically, we aimed to prepare novel iodoarenes that could potentially form helical structures in solution in a similar manner to lactates $\mathbf{1}$ and $\mathbf{2}$, as shown by Muñiz. ${ }^{13}$ It is known that oligomeric ureas can adopt helical conformations, ${ }^{14,15}$ therefore we designed a small family of iodoarenes containing urea appendages (Fig. 2) ${ }^{16}$ It was anticipated that ureas $\mathbf{1 3}$ would be readily prepared from the isocyanate $\mathbf{1 4}$, itself derived from 2-iodoaniline 15. In a similar manner, the $C_{2}$ symmetric bisureas 16 would be obtained from isocyanates 18, which could be prepared from bisanilines $\mathbf{1 8}$ or dicarboxylic acids $\mathbf{1 9}$. We knew that these ureas would not display helicity, as a chain of at least four ureas would be required, but this was our starting point.

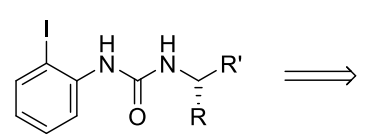

13<smiles>[R]C([R])NC(=O)Nc1cccc(NC(=O)NC([R])[R])c1I</smiles>

16<smiles>O=C(O)c1cccc(C(=O)O)c1I</smiles>

19

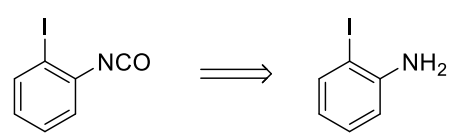

14
Fig. 2. Retrosynthesis of novel chiral iodoarenes 13 and 16 containing urea appendages

In the event, 2-iodoaniline $\mathbf{1 5}$ was readily converted into isocyanate $\mathbf{1 4}$, by the literature procedure, ${ }^{17}$ and this was efficiently transformed into ureas $\mathbf{1 3 a}$ and $\mathbf{1 3 b}$ in very good yields under typical reaction conditions (Scheme 3 ).

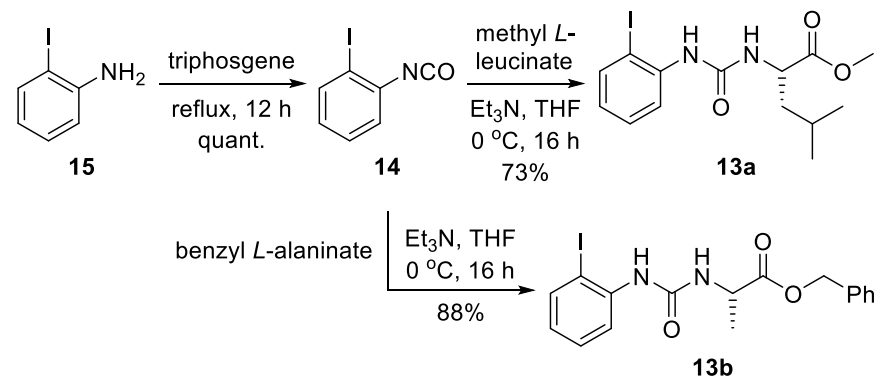

Scheme 3. Preparation of ureas 13a and 13b

Delighted by this success, we endeavored to prepare bisaniline 18 by an identical, but two-directional, two-step protocol starting from commercially available 2,6-dinitroaniline $\mathbf{2 0}$ (Scheme 4). Initial conversion of the amino group to an iodide through diazotization proceeded to give $\mathbf{2 1}$ in good yield, ${ }^{18}$ however subsequent attempts to reduce the two nitro groups were unsuccessful. Treatment with Fe powder (under a variety of conditions), $\mathrm{H}_{2}$ gas or hydrazine over $\mathrm{Pd}$ on charcoal, and trichlorosilane were all ineffective. In all cases studied, partial reductions and/or side reactions including deiodination were observed.<smiles></smiles>

Scheme 4. Initial attempts to prepare bisaniline $\mathbf{1 8}$

Accordingly, we turned our attention to the second route starting from 2-aminoisophthalic acid 22; this was converted readily into iodide 19 in $80 \%$ yield (Scheme 5 ).${ }^{19}$ Conversion of the carboxylic acid directly to the isocyanate 17 using diphenylphosphoroyl azide was unsuccessful; however, successive conversion to the acid chloride and the azide 24 led to the isocyanate $\mathbf{1 7}$ in quantitative yield by a Curtius rearrangement. 
<smiles>Nc1c(C(=O)O)cccc1C(=O)O</smiles>

22

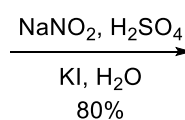

$80 \%$<smiles>O=[N+]([O-])c1cccc([N+](=O)[O-])c1I</smiles>

$\frac{\text { PhMe, reflux, } 2 \mathrm{~h}}{96 \% \text { from diacid } 19}$

17<smiles>O=C(O)c1cccc(C(=O)O)c1I</smiles>

\begin{tabular}{l|l} 
1. $\mathrm{SOCl}_{2}$ & 2. $\mathrm{NaN}_{3}$
\end{tabular} reflux, $2 \mathrm{~h}$, THF, $\mathrm{H}_{2} \mathrm{O}, 2 \mathrm{~h}$<smiles>NC(=O)c1cccc(C(N)=O)c1I</smiles>
24
Scheme 5. Successful route to bisisocyanate $\mathbf{1 7}$

With the isocyanate $\mathbf{1 7}$ in hand, a variety of bisureas were prepared by addition of amines in THF (Scheme 6). A range of amino alcohols were used to generate ureas 16a-16e, which carry primary or secondary alcohols apparently ready for further derivatization. 16f has terminal carboxylic acid groups prone for further functionalization. Finally, 16g is the benzoyl ester of 16c, although it was prepared by the coupling reaction shown rather than esterification.

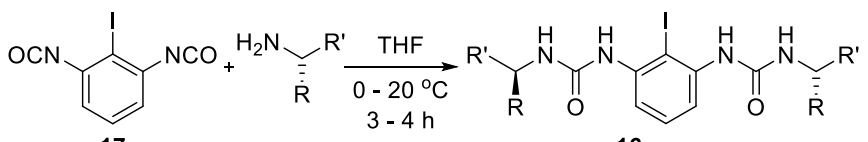
17 16<smiles>CCC(CO)NC(=O)Nc1cccc(NC(=O)Nc2cccc(NC(=O)NC(C)CO)c2C)c1C</smiles>

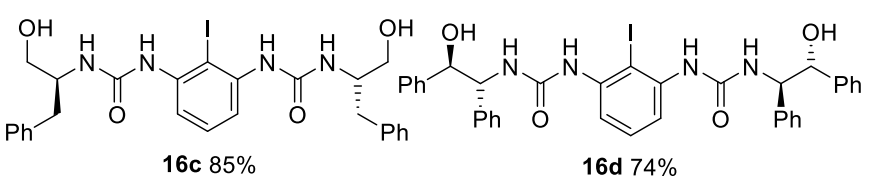<smiles>Cc1ccccc1NC(=O)Nc1cccc(NC(=O)N[C@H]2Cc3ccccc3[C@@H]2O)c1I</smiles>

$16 \mathrm{e} 63 \%$<smiles>O=C(Nc1cccc(NC(=O)N[C@H](Cc2ccccc2)C(=O)O)c1I)NC(C(=O)O)c1ccccc1</smiles><smiles>O=C(Nc1cccc(NC(=O)NC(Cc2ccccc2)Cc2ccccc2)c1I)NC(COC(=O)c1ccccc1)COC(=O)c1ccccc1</smiles>

Scheme 6. Preparation of bisureas 16

Attempts to convert bisurea $\mathbf{1 6 c}$ into tetraurea 25 via conversion of the primary alcohols into tosylates or primary amines were unsuccessful (Scheme 7). In both cases, mixtures of unknown compounds were formed. Similarly, attempts to convert the carboxylic acids in $\mathbf{1 6 f}$ into amides via acid chloride formation were unproductive. These ureas 16a-f exhibit low solubility in organic solvents, which could explain the difficulties faced in their synthetic manipulation.

$16 c$

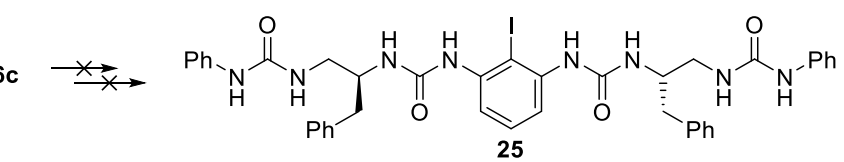

Scheme 7. Unsuccessful attempts to prepare tetraurea 25
Next, we prepared alcohol 28, which contained a urea functionality, and coupled this with bisisocyanate 17 (Scheme 8). The desired product 29, which contained two urethanes and two ureas, was isolated in good yield.

$$
\text { quant. }
$$

Scheme 8. Preparation of mixed urethane-urea 29

The ability of these 10 novel urea-containing chiral iodoarenes to effect the enantioselective dearomatizing cyclization of naphthyl amide 11a was investigated (Table 1). A subset of the experiments undertaken are presented but it can be seen that products were typically obtained in low yields, and that they could not be purified sufficiently for HPLC analysis. Using iodoarene 16c did lead to pure samples of 12a being isolated, however selectivity was very low (entries 7-9). Using polar solvents like $\mathrm{EtOH}, \mathrm{MeOH}$ and HFIP often leads to higher yields of product but poorer levels of selectivity in hypervalent iodine mediated reactions. ${ }^{9,20}$ The highest yield of product was obtained with precatalyst 29 , but the selectivity was just $2 \%$ ee. It is likely that the active catalytic species, i.e. the iodine(III) compounds, are even more sparingly soluble than the parent iodoarenes, which could explain their low reactivities. 
Table 1. Efficacy of novel urea-based iodoarenes in the dearomatizing cyclization of naphthyl amide 11a

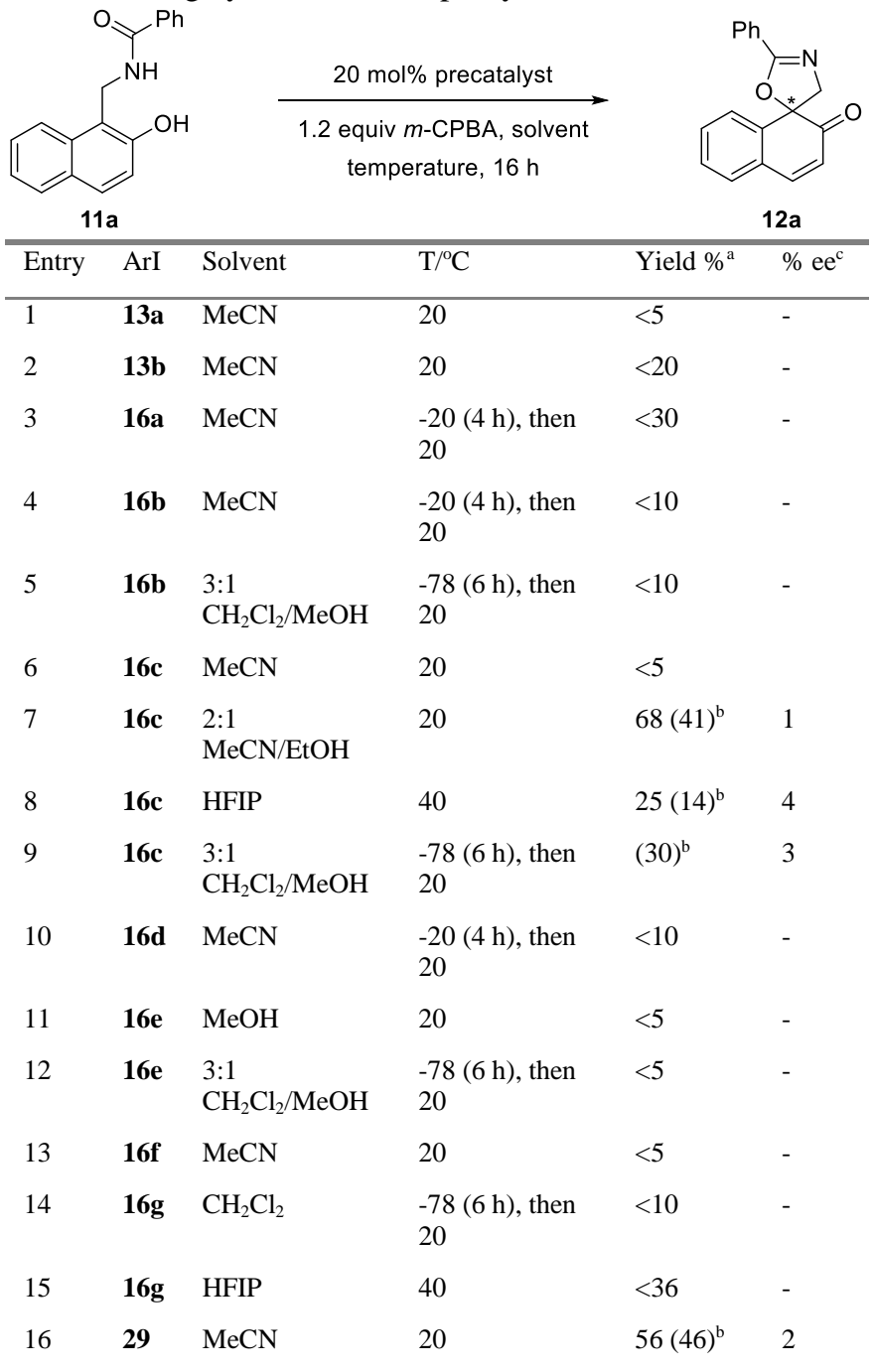

${ }^{\mathrm{a}}$ Yield determined by ${ }^{1} \mathrm{H}$ NMR using 1,3,5-trimethoxybenzene as an internal standard. ${ }^{b}$ Yield of pure isolated compound. ${ }^{\mathrm{c}}$ Determined by chiral HPLC analysis.

In summary, we have developed a synthetic route into ureabased chiral iodoarenes. In particular, a two-directional strategy to symmetrical bisureas has been revealed. Some of these compounds can effectively catalyze the dearomatizing cyclization of a naphthyl amide. Unfortunately, these precatalysts were not effective in delivering the dearomatizing cyclization products in high enantioselectivities, but they may be useful in other iodine(I/III)-catalyzed processes.

\section{Experimental section}

\subsection{General}

Infrared (IR) spectra were recorded on a Nicolet 380, equipped with a diamond probe ATR attachment. Low and high resolution mass spectra $(\mathrm{m} / \mathrm{z})$ were obtained in the electrospray (ESI) mode. Melting points (uncorrected) were measured on a Stuart SMP10 apparatus. All reagents and solvents were used without further purification except THF (dried over $3 \AA$ MS and then distilled over $\mathrm{Na}$ /benzophenone under $\mathrm{N}_{2}$ ). The reactions were monitored by thin layer chromatography (TLC) using F254 pre-coated silica gel plates. Spots were visualized with $\mathrm{UV}, \mathrm{KMnO}_{4}$ stain or vanillin stain. Flash chromatography was performed with $35-70 \mu \mathrm{m}, 60 \AA$ silica gel.

\subsection{Synthesis of 2'-phenyl-2H,4'H-spiro[naphthalene-1,5'- oxazol]-2-one $(12 a)$}

To a solution of amide 11a (1 equiv) in anhydrous solvent $(0.030 \mathrm{M})$ at room temperature (or below) was added $m$-CPBA ( 2.2 equiv) and iodoarene catalyst ( 0.1 or 0.2 equiv). The reaction mixture was stirred for $16 \mathrm{~h}$ and then quenched with a saturated solution of $\mathrm{NaHCO}_{3}$. The organic layer was extracted with ethyl acetate (three times), dried over $\mathrm{MgSO}_{4}$, filtered and concentrated under vacuum. The resulting residue was purified by flash chromatography (3:1 petroleum ether/EtOAc) to afford 12a as a pale yellow oil. IR (thin film) 3160, 3053, 2869, 1715, 1673, 1652, $1603,1569,1547,1367,1338,1283,770 \mathrm{~cm}^{-1} ;{ }^{1} \mathrm{H}$ NMR $\left(\mathrm{CDCl}_{3}\right.$, $400 \mathrm{MHz})$ 8.08-8.06 (m, 2H), 7.56-7.35 (m, 8H), $6.21(\mathrm{~d}, J=10.0$ $\mathrm{Hz}, 1 \mathrm{H}), 4.48(\mathrm{~d}, J=15.4 \mathrm{~Hz}, 1 \mathrm{H}), 4.01(\mathrm{~d}, J=15.4 \mathrm{~Hz}, 1 \mathrm{H}) ;{ }^{13} \mathrm{C}$ NMR $\left(\mathrm{CDCl}_{3} 100 \mathrm{MHz}\right)$ 197.8, 164.3, 145.8, 142.3, 132.0, 131.0, 129.7, 129.0, 128.9, 128.8, 128.6, 127.0, 125.7, 123.7, 86.6, 69.8; HRMS $[\mathrm{M}+\mathrm{H}]^{+} \mathrm{m} / \mathrm{z}$ calc'd for $\mathrm{C}_{18} \mathrm{H}_{14} \mathrm{NO}_{2}$ requires 276.1019, found 276.1019. HPLC: Chiralpak IB, $254 \mathrm{~nm}$, hexane/IPA gradient (100:0 to $90: 10$ over $35 \mathrm{~min}$ ), $1 \mathrm{~mL} / \mathrm{min}$, retention times 12.9 \& 15.1 minutes.

\subsection{Preparation of ureas 13}

To the solution of 1-iodo-2-isocyanatobenzene 14 (0.50 g, 2.0 mmol, 1 equiv) in THF $(30 \mathrm{~mL})$ at $0{ }^{\circ} \mathrm{C}$ was added a solution of amino ester ( $2.2 \mathrm{mmol}, 1.1$ equiv) and triethylamine $(0.57 \mathrm{~mL}, 4.1$ mmol, 2.0 equiv) in THF $(10 \mathrm{~mL})$ dropwise. Then, the ice bath was removed and the reaction mixture was stirred overnight. The reaction mixture was concentrated under reduced pressure was washed with hexanes multiple times. Recrystallization either with dichloromethane/ethyl acetate or ethanol provided the urea 13.

\subsubsection{Methyl ((2-iodophenyl)carbamoyl)-L-} leucinate (13a)

Yellow solid (0.58 g, 73\%). M.p. $158-160{ }^{\circ} \mathrm{C}$; IR 3301, 2951, 2868, 1737, 1641, 1573, 1557, 1519, 1463, 1433, 1367, 1273, 1255, 1205, 1155, $1016 \mathrm{~cm}^{-1}$; ${ }^{1} \mathrm{H}$ NMR (DMSO-d $d^{6}, 400 \mathrm{MHz}$ ) 7.84-7.78 (m, 2H), $7.72(\mathrm{~s}, 1 \mathrm{H}), 7.51(\mathrm{~d}, J=7.5 \mathrm{~Hz}, 1 \mathrm{H}), 7.28(\mathrm{t}$, $J=8.1 \mathrm{~Hz}, 1 \mathrm{H}), 6.76(\mathrm{t}, J=7.5 \mathrm{~Hz}, 1 \mathrm{H}), 4.26-4.21(\mathrm{~m}, 1 \mathrm{H}), 3.65$ $(\mathrm{s}, 3 \mathrm{H}), 1.73-1.66(\mathrm{~m}, 1 \mathrm{H}), 1.58(\mathrm{t}, J=7.3 \mathrm{~Hz}, 2 \mathrm{H}), 0.93(\mathrm{~d}, J=$ $6.5 \mathrm{~Hz}, 3 \mathrm{H}), 0.89$ (d, $J=6.5 \mathrm{~Hz}, 3 \mathrm{H}) ;{ }^{13} \mathrm{C}$ NMR (DMSO- $d^{6}, 100$ MHz) 173.7, 154.7, 140.3, 138.9, 128.5, 124.3, 122.0, 90.0, 51.8, 51.0, 40.6, 24.3, 22.7, 21.5; HRMS $[\mathrm{M}+\mathrm{H}]^{+} \mathrm{m} / \mathrm{z}$ calc'd for $\mathrm{C}_{14} \mathrm{H}_{20} \mathrm{IN}_{2} \mathrm{O}_{3}$ 391.0513, found 391.0510.

\subsubsection{Benzyl ((2-iodophenyl)carbamoyl)-L- alaninate $(\mathbf{1 3 b})$}

Pale yellow solid (0.37 g, 88\%). M.p. $161-163{ }^{\circ} \mathrm{C}$; IR 3299, 3060, 2979, 2605, 2498, 1720, 1633, 1556, 1512, 1463, 1452, 1432, 1318, 1291, 1221, 1164, $1097 \mathrm{~cm}^{-1}$; ${ }^{1} \mathrm{H}$ NMR (DMSO- $d^{6}$, $400 \mathrm{MHz}) 7.84-7.79(\mathrm{~m}, 2 \mathrm{H}), 7.74(\mathrm{~s}, 1 \mathrm{H}), 7.58(\mathrm{~d}, J=6.9 \mathrm{~Hz}$, 1H), 7.39-7.27 (m, 6H), $6.77(\mathrm{t}, J=7.4 \mathrm{~Hz}, 1 \mathrm{H}), 5.20-5.11(\mathrm{~m}$, $2 \mathrm{H}), 4.33-4.24(\mathrm{~m}, 1 \mathrm{H}), 1.35(\mathrm{~d}, J=7.4 \mathrm{~Hz}, 3 \mathrm{H}) ;{ }^{13} \mathrm{C} \mathrm{NMR}$ (DMSO-d6, $100 \mathrm{MHz}$ ) 173.2, 154.5, 140.3, 138.9, 136.1, 128.5, 128.5, 128.0, 127.7, 124.4, 122.1, 90.1, 65.9, 48.5, 17.6; HRMS $[\mathrm{M}+\mathrm{H}]^{+} \mathrm{m} / z$, calc'd for $\mathrm{C}_{17} \mathrm{H}_{18} \mathrm{IN}_{2} \mathrm{O}_{3} 425.0357$, found 425.0359 .

\subsection{Preparation of 2-iodo-1,3-diisocyanatobenzene 17}

A solution of 2-iodoisophthalic acid 19 (1.5 g, $5.1 \mathrm{mmol})$ in thionyl chloride $(40 \mathrm{~mL})$ was heated to reflux for $2 \mathrm{~h}$ then concentrated under reduced pressure to afford 2-iodoisophthaloyl chloride. This crude mixture was dissolved in THF $(15 \mathrm{~mL})$, cooled to $0{ }^{\circ} \mathrm{C}$ and $\mathrm{NaN}_{3}(2.2 \mathrm{~g}, 34 \mathrm{mmol})$ dissolved in water $(8$ 
$\mathrm{mL}$ ) was added. After $2 \mathrm{~h}$, the reaction mixture was quenched with saturated aqueous $\mathrm{NaHCO}_{3}(10 \mathrm{~mL})$ solution and extracted with toluene $(30 \mathrm{~mL})$. This was dried over $\mathrm{MgSO}_{4}$ and evaporated to half under reduced pressure to give a toluene solution of 2iodoisophthaloyl diazide 24. Without further purification, the crude mixture was heated at reflux for 2 hours and then concentrated to $\sim 3 \mathrm{~mL}$ to afford $\mathbf{1 7}$ as a light brown solution (1.42 g, $96 \%$ - determined by NMR). The progress of the reaction was monitored by IR. IR 3370, 3024, 2251, 2141, 1723, 1573, 1494, 1396, 1214, 781, $728 \mathrm{~cm}^{-1} ;{ }^{1} \mathrm{H} \mathrm{NMR}\left(\mathrm{CDCl}_{3}, 400 \mathrm{MHz}\right) 7.20-7.30$ $(\mathrm{m}, 1 \mathrm{H}), 6.93(\mathrm{~d}, J=8.0 \mathrm{~Hz}, 2 \mathrm{H}) ;{ }^{13} \mathrm{C} \mathrm{NMR}\left(\mathrm{CDCl}_{3}, 100 \mathrm{MHz}\right)$ 138.2, 130.0, 129.8, 122.2, 97.2; HRMS $[\mathrm{M}-\mathrm{CO}+3 \mathrm{H}]^{+} \mathrm{m} / \mathrm{z}$ calc'd for $\mathrm{C}_{7} \mathrm{H}_{6} \mathrm{IN}_{2} \mathrm{O} 260.9519$, found 260.9516 .

\subsection{Preparation of bisureas 16}

To a solution of 2-iodo-1,3-diisocyanatobenzene $17(0.70 \mathrm{~mL}$, $4.3 \mathrm{mmol}, 1$ equiv) in THF $(60 \mathrm{~mL})$ at $0{ }^{\circ} \mathrm{C}$ was added dropwise a solution of amine (1.5 g, $9.5 \mathrm{mmol}, 2.2$ equiv) in THF $(10 \mathrm{~mL})$. The ice bath was removed and the reaction mixture was stirred for 3-4 hours or until TLC analysis showed completion of the reaction. Then, the reaction mixture was concentrated under reduced pressure and the residue washed with hexanes multiple times. Recrystallization from ethyl acetate and ethanol provided the bisurea 16. Typically, solvents could not be completely removed from these compounds.

\subsubsection{1,1'-(2-iodo-1,3-phenylene ) bis(3-((S)-1-} hydroxypropan-2-yl)urea)(16a)

White solid. (0.50 g, 81\%). M.p. 249-250 ${ }^{\circ} \mathrm{C}$; IR 3284, 2980, $2851,1632,1162,1035 \mathrm{~cm}^{-1} ;{ }^{1} \mathrm{H}$ NMR (DMSO- $\left.d^{6}, 400 \mathrm{MHz}\right) 7.60$ $(\mathrm{s}, 2 \mathrm{H}), 7.38(\mathrm{~d}, J=7.9 \mathrm{~Hz}, 2 \mathrm{H}), 7.14(\mathrm{t}, J=8.1 \mathrm{~Hz}, 1 \mathrm{H}), 6.89(\mathrm{~d}$, $J=7.6 \mathrm{~Hz}, 2 \mathrm{H}$ ), 4.77 (br s, 2H), 3.70-3.65 (m, 2H), 3.42-3.28 (m, $4 \mathrm{H}), 1.08(\mathrm{~d}, J=6.8 \mathrm{~Hz}, 6 \mathrm{H}) ;{ }^{13} \mathrm{C}$ NMR (DMSO- $\left.d^{6}, 100 \mathrm{MHz}\right)$ 154.7, 141.2, 127.8, 117.1, 89.3, 64.8, 47.0, 17.8; HRMS [M+H] ${ }^{+}$ $\mathrm{m} / z$ calc'd for $\mathrm{C}_{14} \mathrm{H}_{22} \mathrm{IN}_{4} \mathrm{O}_{4} 437.0680$, found 437.0698 .

\subsubsection{1,1'-(2-iodo-1,3-phenylene ) bis(3-((S)-1-}

hydroxybutan-2-yl)urea) (16b)

Pale yellow solid (0.60 g, 90\%). M.p. $252-254{ }^{\circ} \mathrm{C}$; IR 3290, 2962, 2930, 2873, 2355, 1633, 1556, 1463, 1410, 1274, 1223, 1074, $1018 \mathrm{~cm}^{-1}$; ${ }^{1} \mathrm{H}$ NMR (DMSO- $\left.d^{6}, 400 \mathrm{MHz}\right) 7.62(\mathrm{~s}, 2 \mathrm{H})$, $7.38(\mathrm{~d}, J=8.1 \mathrm{~Hz}, 2 \mathrm{H}), 7.13(\mathrm{t}, J=8.1 \mathrm{~Hz}, 1 \mathrm{H}), 6.84(\mathrm{~d}, J=8.1$ $\mathrm{Hz}, 2 \mathrm{H}), 4.72$ (br s, 2H), 3.56-3.42 (m, 4H), 3.35-3.32 (m, 2H), $1.64-1.54(\mathrm{~m}, 2 \mathrm{H}), 1.43-1.31(\mathrm{~m}, 2 \mathrm{H}), 0.89(\mathrm{t}, J=7.4 \mathrm{~Hz}, 6 \mathrm{H}) ;{ }^{13} \mathrm{C}$ NMR (DMSO- $\left.d^{6}, 100 \mathrm{MHz}\right) 155.0,141.3,127.8,117.1,89.3$, 63.0, 52.5, 24.2, 10.5; HRMS $[\mathrm{M}+\mathrm{H}]^{+} \mathrm{m} / z$ calc'd for $\mathrm{C}_{16} \mathrm{H}_{26} \mathrm{IN}_{4} \mathrm{O}_{4}$ 465.0993 , found 465.0990 .

\subsubsection{1, 1'-(2-iodo-1,3-phenylene ) bis(3-((S)-1-}

hydroxy-3-phenylpropan-2-yl)urea) (16c)

Yellow solid (1.7 g, 85\%). M.p. 265-266 ${ }^{\circ} \mathrm{C}$; IR 3297, 3024, 2919, 1634, 1548, 1465, $1271 \mathrm{~cm}^{-1} ;{ }^{1} \mathrm{H}$ NMR (DMSO- $d^{6}, 400$ MHz) 7.64 (s, 2H), 7.32-7.20 (m, 12H), $7.10(\mathrm{t}, J=8.1 \mathrm{~Hz}, 1 \mathrm{H})$, $6.98(\mathrm{~d}, J=8.1 \mathrm{~Hz}, 2 \mathrm{H}), 4.90(\mathrm{br} \mathrm{s}, 2 \mathrm{H}), 3.85-3.78(\mathrm{~m}, 2 \mathrm{H}), 3.43-$ $3.32(\mathrm{~m}, 4 \mathrm{H}), 2.91-2.81(\mathrm{~m}, 2 \mathrm{H}), 2.73-2.65(\mathrm{~m}, 2 \mathrm{H}) ;{ }^{13} \mathrm{C}$ NMR (DMSO- $\left.d^{6}, 100 \mathrm{MHz}\right) 154.7,141.2,139.2,129.3,128.2,127.7$, $125.9,117.2,89.5,62.4,52.8,37.3$; HRMS $[\mathrm{M}+\mathrm{H}]^{+} \mathrm{m} / \mathrm{z}$ calc'd for $\mathrm{C}_{26} \mathrm{H}_{30} \mathrm{IN}_{4} \mathrm{O}_{4}$ 589.1306, found 589.1321.

3.5.4. 1,1'-(2-iodo-1,3-phenylene)bis(3-((1S,2R)-2hydroxy-1,2-diphenylethyl)urea) (16d)

White solid (370 mg, 74\%). M.p. 221-223 ${ }^{\circ} \mathrm{C}$; IR 3343, 3213, 2981, 1647, 1587, 1556, 1503, 1465, 1396, 1276, 1231, 1068, $1047 \mathrm{~cm}^{-1} ;{ }^{1} \mathrm{H}$ NMR (DMSO-d $\left.d^{6}, 400 \mathrm{MHz}\right) 7.98$ (s, 2H), 7.67 (d, $J=8.8 \mathrm{~Hz}, 2 \mathrm{H}), 7.47(\mathrm{~d}, J=7.4 \mathrm{~Hz}, 4 \mathrm{H}), 7.42(\mathrm{~d}, J=7.4 \mathrm{~Hz}, 4 \mathrm{H})$, 7.36-7.32 (m, 8H), 7.26-7.20 (m, 4H), 7.07-7.06 (m, 2H), 6.99$6.93(\mathrm{~m}, 1 \mathrm{H}), 5.67(\mathrm{~d}, J=4.1 \mathrm{~Hz}, 2 \mathrm{H}), 4.90-4.83(\mathrm{~m}, 4 \mathrm{H}) ;{ }^{13} \mathrm{C}$
NMR (DMSO- $\left.d^{6}, 100 \mathrm{MHz}\right) 154.7,143.7,142.8,141.1,127.8$, 127.6, 127.4, 127.1, 126.8, 126.6, 126.4, 117.6, 90.0, 75.6, 59.1; HRMS [M+Na] ${ }^{+} \mathrm{m} / z$ calc' $d$ for $\mathrm{C}_{36} \mathrm{H}_{33} \mathrm{IN}_{4} \mathrm{O}_{4} \mathrm{Na} 735.1439$, found 735.1441 .

3.5.5. 1,1'-(2-iodo-1,3-phenylene)bis(3-((1R,2S)-1hydroxy-2,3-dihydro-1H-inden-2-yl)urea) (16e)

Off-white solid (0.52 g, 63\%). M.p. $247-248^{\circ} \mathrm{C}$; IR 3318, 3240, 2912, 1682, 1632, 1550, 1465, 1400, 1359, 1231, 1142, $1047 \mathrm{~cm}^{-}$ ${ }^{1}$; ${ }^{1} \mathrm{H}$ NMR (DMSO-d $\left.d^{6}, 400 \mathrm{MHz}\right) 8.09$ (s, 2H), 7.46 (d, $J=8.1$ $\mathrm{Hz}, 2 \mathrm{H}), 7.29-7.18(\mathrm{~m}, 11 \mathrm{H}), 5.23(\mathrm{~d}, J=4.1 \mathrm{~Hz}, 2 \mathrm{H}), 5.11(\mathrm{dd}, J$ $=8.7,4.9 \mathrm{~Hz}, 2 \mathrm{H}), 4.47(\mathrm{q}, J=4.5 \mathrm{~Hz}, 2 \mathrm{H}), 3.07(\mathrm{dd}, J=16.2,4.6$ $\mathrm{Hz}, 2 \mathrm{H}), 2.81\left(\mathrm{~d}, J=16.1 \mathrm{~Hz}, 2 \mathrm{H}\right.$ ); ${ }^{13} \mathrm{C}$ NMR (DMSO- $d^{6}, 100$ $\mathrm{MHz}) 155.5,143.2,141.4,140.5,127.8,127.1,126.3,124.9$, 124.1, 118.3, 90.9, 72.3, 57.6; HRMS $[\mathrm{M}+\mathrm{H}]^{+} \mathrm{m} / \mathrm{z}$ calc'd for $\mathrm{C}_{26} \mathrm{H}_{26} \mathrm{IN}_{4} \mathrm{O}_{4}$ 585.0993, found 585.0989.

3.5.6. (2S, 2'S)-2, ''-(( ( 2 -iodo-1,3-

phenylene)bis(azanediyl))bis(carbonyl))bis(azanedi $y l)$ )bis(3-phenylpropanoic acid) (16f)

Pale-yellow solid (0.76 g, 70\%). M.p. 227-229 ${ }^{\circ}$ C; IR 3217, 3025, 2890, 1714, 1638, 1547, 1494, 1464, 1407, 1275, 1214, $1075 \mathrm{~cm}^{-1}$; ${ }^{1} \mathrm{H}$ NMR (DMSO-d $\left.d^{6}, 400 \mathrm{MHz}\right) 7.88$ (s, 2H), 7.33-7.21 $(\mathrm{m}, 16 \mathrm{H}), 7.15-7.11(\mathrm{~m}, 1 \mathrm{H}), 4.48-4.40(\mathrm{~m}, 2 \mathrm{H}), 3.14-3.07(\mathrm{~m}$, 2H), 2.98-2.90 (m, 2H); ${ }^{13} \mathrm{C}$ NMR (DMSO- $\left.d^{6}, 100 \mathrm{MHz}\right) 173.5$, 154.6, 141.0, 137.3, 136.6, 129.5, 128.5, 126.5, 117.8, 90.1, 54.0, 37.6; HRMS: $[\mathrm{M}+\mathrm{H}]^{+} \mathrm{m} / z$ calc'd for $\mathrm{C}_{26} \mathrm{H}_{26} \mathrm{IN}_{4} \mathrm{O}_{6} 617.0892$, found 617.0898

\subsection{7. (2S,2'S)-((((2-iodo-1,3}

phenylene)bis(azanediyl))bis(carbonyl))bis(azanedi yl))bis(3-phenylpropane-2,1-diyl) dibenzoate (16g)

Yellow solid (120 mg, 49\%). M.p. 230-232 ${ }^{\circ} \mathrm{C}$; IR 3288, 3064, 2923, 2852, 1716, 1641, $1538 \mathrm{~cm}^{-1}$; ${ }^{1} \mathrm{H}$ NMR (DMSO-d $d^{6}, 400$ $\mathrm{MHz})$ 8.05-8.02 (m, 4H), 7.70-7.66 (m, 2H), 7.57-7.52 (m, 4H), 7.41-7.37 (m, 2H), 7.33-7.30 (m, 10H), 7.26-7.21 (m, 4H), 7.14$7.08(\mathrm{~m}, 1 \mathrm{H}), 4.36-4.17(\mathrm{~m}, 6 \mathrm{H}), 2.99-2.84(\mathrm{~m}, 4 \mathrm{H}) ;{ }^{13} \mathrm{C} \mathrm{NMR}$ (DMSO- $d^{6}$, $\left.100 \mathrm{MHz}\right) 165.7,154.7,149.2,138.2,133.5,129.7$, 129.4, 129.2, 128.8, 128.4, 128.0, 126.3, 119.3, 87.9, 66.1, 49.8, 37.4; HRMS $[\mathrm{M}+\mathrm{H}]^{+} \mathrm{m} / z$ calc'd for $\mathrm{C}_{40} \mathrm{H}_{38} \mathrm{IN}_{4} \mathrm{O}_{6}$ 797.1831, found 797.1835 .

\subsection{Preparation of 1-((S)-1-hydroxy-3-phenylpropan-2-yl)-3-} ((S)-1-phenylpropyl)urea 28

To a solution of $(S)$-(1-isocyanatopropyl)benzene $26(0.50 \mathrm{~g}$, $3.1 \mathrm{mmol}, 1$ equiv) in THF $(40 \mathrm{~mL})$ at $0{ }^{\circ} \mathrm{C}$ was added dropwise a solution of amine 27 (0.52 g, $3.4 \mathrm{mmol}, 1.1$ equiv) in THF (10 $\mathrm{mL})$. The ice bath was removed and the reaction mixture was stirred for 4 hours. The reaction mixture was concentrated under reduced pressure and the residue was washed with hexanes multiple times and then purified by flash chromatography (silica gel; $1: 1$ petroleum ether/EtOAc) to provide $\mathbf{2 8}$ as a white solid (1.0 g, 99\%). M.p. $130-131{ }^{\circ} \mathrm{C}$; IR 3336, 3026, 2927, 2356, 2341, 1610, 1586, 1565, 1493, 1449, 1380, 1253, 1073, $1046 \mathrm{~cm}^{-1} ;{ }^{1} \mathrm{H}$ NMR (DMSO- $\left.d^{6}, 400 \mathrm{MHz}\right) 7.32-7.14(\mathrm{~m}, 10 \mathrm{H}), 6.40(\mathrm{~d}, J=8.6 \mathrm{~Hz}$, $1 \mathrm{H}), 5.79(\mathrm{~d}, J=8.6 \mathrm{~Hz}, 1 \mathrm{H}), 4.83(\mathrm{br} \mathrm{s}, 1 \mathrm{H}), 4.50(\mathrm{q}, J=7.3 \mathrm{~Hz}$, $1 \mathrm{H}), 3.71-3.67(\mathrm{~m}, 1 \mathrm{H}), 3.35-3.25(\mathrm{~m}, 2 \mathrm{H}), 2.80-2.75(\mathrm{~m}, 1 \mathrm{H})$, 2.62-2.57 (m, 1H), 1.67-1.53 (m, 2H), $0.80(\mathrm{t}, J=7.3 \mathrm{~Hz}, 3 \mathrm{H}) ;{ }^{13} \mathrm{C}$ NMR (DMSO- $\left.d^{6}, 100 \mathrm{MHz}\right) 157.2,144.6,139.2,129.2,128.1$, $128.1,126.2,62.5,54.3,52.4,37.2,30.0,10.6$; HRMS [M+H] ${ }^{+}$ $\mathrm{m} / \mathrm{z}$ calc'd for $\mathrm{C}_{19} \mathrm{H}_{25} \mathrm{~N}_{2} \mathrm{O}_{2}$ 313.1911, found 313.1909.

\subsection{Preparation of bis((S)-3-phenyl-2-(3-((S)-1- phenylpropyl)ureido)propyl)(2-iodo-1,3-phenylene)dicarbamate 29}

2-Iodo-1,3-diisocyanatobenzene 17 ( $350 \mathrm{mg}, 1.2 \mathrm{mmol}, 1$ equiv - as a solution in toluene) and triethylamine $(0.37 \mathrm{~mL}, 2.7 \mathrm{mmol}$, 
2.2 equiv) were dissolved in dichloromethane $(30 \mathrm{~mL})$ and cooled to $0{ }^{\circ} \mathrm{C}$ under $\mathrm{N}_{2}$. Urea $28(0.75 \mathrm{~g}, 2.4 \mathrm{mmol}, 2.2$ equiv) was dissolved in dichloromethane $(20 \mathrm{~mL})$ in a separate flask and added to the first solution dropwise over 10 minutes. The reaction mixture was stirred overnight at room temperature and then concentrated under reduced pressure. The residue was quenched with $1 \mathrm{~N} \mathrm{HCl}(20 \mathrm{~mL})$ and extracted with EtOAc $(20 \mathrm{~mL} \times 3)$, dried over $\mathrm{MgSO}_{4}$ and concentrated under reduced pressure. Recrystallization from $\mathrm{CH}_{2} \mathrm{Cl}_{2} / \mathrm{EtOAc}$ afforded 29 as a dark brown solid (0.73 g, 65\%). M.p. 249-251 ${ }^{\circ}$ C; IR 3270, 3026, 2961, 1702, $1633,1584,1519,1461,1398,1202,1109,1109,1018 \mathrm{~cm}^{-1} ;{ }^{1} \mathrm{H}$ NMR (DMSO- $\left.d^{6}, 400 \mathrm{MHz}\right) 9.01(\mathrm{~s}, 2 \mathrm{H}), 7.32-7.17(\mathrm{~m}, 23 \mathrm{H})$, $6.43(\mathrm{~d}, J=8.0 \mathrm{~Hz}, 2 \mathrm{H}), 5.86(\mathrm{~d}, J=8.0 \mathrm{~Hz}, 2 \mathrm{H}), 4.56-4.49(\mathrm{~m}$, $2 \mathrm{H}), 4.02-3.95(\mathrm{~m}, 6 \mathrm{H}), 2.83-2.70(\mathrm{~m}, 4 \mathrm{H}), 1.67-1.59(\mathrm{~m}, 4 \mathrm{H})$, $0.79(\mathrm{t}, J=7.0 \mathrm{~Hz}, 6 \mathrm{H}) ;{ }^{13} \mathrm{C}$ NMR (DMSO- $\left.d^{6}, 100 \mathrm{MHz}\right) 157.0$, $154.2,144.5,140.6,138.2,129.3,128.5,128.5,128.1,126.4$, 126.2 , 83.2, 65.8, 54.4, 49.8, 37.3, 29.9, 10.7; HRMS [M+Na] ${ }^{+} \mathrm{m} / \mathrm{z}$ calc'd for $\mathrm{C}_{46} \mathrm{H}_{51} \mathrm{IN}_{6} \mathrm{NaO}_{6}$ 933.2807, found 933.2813.

\section{Acknowledgments}

We thank the University of Huddersfield for funding (feewaiver scholarship to MUT). We thank Mirdyul Das (University of Huddersfield) for the preparation of iodoarene 1a.

\section{References and notes}

1. For reviews, see: (a) Parra, A. Chem. Rev. 2019, 119, 1203312088; (b) Flores, A.; Cots, E.; Bergès, J.; Muñiz, K. Adv. Synth. Catal. 2019, 361, 2-25; (c) Ghosh, S.; Pradhan, S.; Chatterjee, I. Beilstein J. Org. Chem. 2018, 14, 1244-1262; (d) Fujita, M. Tetrahedron Lett. 2017, 58, 4409-4419; (e) Berthiol, F. Synthesis 2015, 47, 587-603; (f) Kumar, R.; Wirth, T. Top. Curr. Chem. 2015, 373, 243-261.

2. Fujita, M.; Yoshida, Y.; Miyata, K.; Wakisaka, A.; Sugimura, T. Angew. Chem. Int. Ed. 2010, 49, 7068-7071.

3. Uyanik, M.; Yasui, T.; Ishihara, K. Angew. Chem. Int. Ed. 2010, 49, 2175-2177.

4. For example, see: (a) Uyanik, M,; Yasui, T.; Ishihara, K. Angew. Chem. Int. Ed. 2013, 52, 9215-9218; (b) Jain, N.; Xu, S.; Ciufolini, M. A. Chem. Eur. J. 2017, 23, 4542-4546.

5. Dohi, T.; Takenaga, N.; Nakae, T.; Toyoda, Y.; Yamasaki, Y.; Shiro, M.; Fujioka, H.; Maruyama, A.; Kita, Y. J. Am. Chem. Soc. 2013, 135, 4558-4566.
6. Antien, K.; Pouységu, L.; Deffieux, D.; Massip, S.; Peixoto, P. A.; Quideau, S. Chem. Eur. J. 2019, 25, 2852-2858.

7. Hashimoto, T.; Shimazaki, Y.; Omatsu, Y.; Maruoka, K. Angew. Chem. Int. Ed. 2018, 57, 7200-7204.

8. Murray, S. J.; Ibrahim, H. Chem. Commun. 2015, 51, 2376-2379.

9. Rodríguez, A.; Moran, W. J. Synthesis 2012, 44, 1178-1182.

10. Abazid, A. H.; Nachtsheim, B. J. Angew. Chem. Int. Ed. 2020, 59, 1479-1484.

11. Tariq, M. U.; Moran, W. J. Eur. J. Org. Chem. 2020, 5153-5160.

12. For the synthesis of iodoarene 1a, see: Banik, S. M.; Medley, J. W.; Jacobsen, E. N. J. Am. Chem. Soc. 2016, 138, 5000-5003.

13. Haubenreisser, S; Wöste, T. H.; Martínez, C.; Ishihara, K.; Muñiz, K. Angew. Chem. Int. Ed. 2016, 55, 413-417.

14. Wechsel, R.; Raftery, J.; Cavagnat, D.; Guichard, G.; Clayden, J. Angew. Chem. Int. Ed. 2016, 55, 9657-9661.

15. Bécart, D.; Diemer, V.; Salaün, A.; Oiarbide, M.; Nelli, Y. R.; Kauffmann, B.; Fischer, L.; Palomo, C.; Guichard, G. J. Am. Chem. Soc. 2017, 139, 12524-12532.

16. For examples of the synthesis of related iodoarenes, see: (a) Gaux, B.; Le Hénaff, P. C. R. Acad. Sc. Serie C 1973, 277, 1033-1035; (b) Hayashi, M.; Sai, H.; Horikawa, H. Heterocycles 1998, 48, 1331-1335; (c) McAtee, L. C.; Sutton, S. W.; Rudolph, D. A.; Li, X.; Aluisio, L. E.; Phuong, V. K.; Dvorak, C. A.; Lovenberg, T. W.; Carruthers, N. I.; Jones, T. K. Bioorg. Med. Chem. Lett. 2004, 14, 4225-4229; (d) Nickerson, D. M.; Mattson, A. E. Chem. Eur. J. 2012, 18, 8310-8314; (e) Wu, J.; Wang, C.; Häberli, C.; White, K. L.; Shackleford, D. M.; Chen, G.; Dong, Y.; Charman, S. A.; Keiser, J.; Vennerstrom, J. L. Bioorg. Med. Chem. Lett. 2018, 28 , 3648-3651; (f) Ruan, B.; Zhang, Y.; Tadesse, S.; Preston, S.; Taki, A. C.; Jabbar, A.; Hofmann, A.; Jiao, Y.; Garcia-Bustos, J.; Harjani, J.; Le, T. G.; Varghese, S.; Teguh, S.; Xie, Y.; Odiba, J.; Hu, M.; Gasser, R. B.; Baell, J. Eur. J. Med. Chem. 2020, 190, 112100.

17. Zeng, F.; Alper, H. Org. Lett. 2010, 12, 3642-3644.

18. Jones, I. M.; Hamilton, A. D. Angew. Chem. Int. Ed. 2011, 50, 4597-4600.

19. Rewcastle, G. W.; Denny, W. A. Synthesis 1985, 217-220.

20. Colomer, I.; Chamberlain, A. E. R.; Haughey, M. B.; Donohoe, T. J. Nat. Rev. Chem. 2017, 1, 0088.

\section{Appendix A. Supplementary Material}

Supplementary data to this article can be found online at 